\title{
Research on Normal Human Plantar Pressure Test
}

\author{
Xi Yang Liu ${ }^{1}$, Ren Jie Gao ${ }^{2 a}$, Si Ling Feng ${ }^{1}$, Xian Mei Chen ${ }^{1}$ and Yao Chi Zhao ${ }^{1}$ \\ ${ }^{1}$ School of College of Information Science and Technology, Hainan university, Hainan Haikou 570228, China \\ ${ }^{2}$ Haikou People's Hospital, Hainan Haikou 570228,China
}

\begin{abstract}
FSR400 pressure sensor, nRF905 wireless transceiver and MSP40 SCM are used to design the insole pressure collection system, LabVIEW is used to make HMI of data acquisition, collecting a certain amount of normal human foot pressure data, statistical analysis of pressure distribution relations about five stages of swing phase during walking, using the grid closeness degree to identify plantar pressure distribution pattern recognition, and the algorithm simulation, experimental results demonstrated this method feasible.
\end{abstract}

\section{Introduction}

When the body in accordance with normal gait, plantar only directly contact with the ground, gait would have plantar pressure. The various factors of human physiology, diseases, etc. will affect the person's gait, it studies the body's normal plantar pressure when walking, that have important reference value for researching gait analysis, medical rehabilitation, clinical and smart shoes, and so on ${ }^{[1]}$.

Based on the physical structure and division of the human foot plantar anatomical region, In accordance with the left and right foot insoles heel, arch, first metatarsal, the second metatarsal, metatarsal 3rd, 4th metatarsal, 5th metatarsal and the first toe area, each placed a film pressure sensor FSR400, each foot collects eight points force ${ }^{[2]}$. Distribution of plantar pressure sensor shown in Figure 1.

\footnotetext{
${ }^{a}$ Corresponding author: Liujinrong98@163.com
}

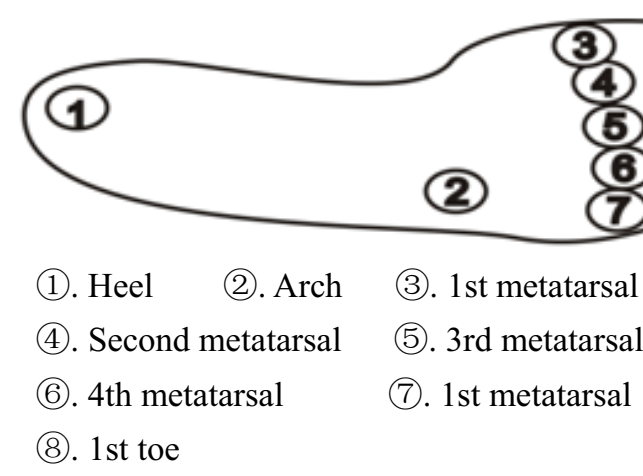

Figure 1. The distribution of plantar pressure sensor

\section{Insole Plantar Pressure Measurement System}

\subsection{Hardware Design of Insole Plantar Pressure Measurement System}

The FSR400 pressure sensor converted the pressure of FSR sensor film region into a change in resistance value, thereby to obtain pressure information, and the greater the pressure, the lower the resistance. The voltage signals of 8 sensors were what we wanted to measure. Because the human body $98 \%$ of plantar pressure signal 
frequencies below $10 \mathrm{~Hz}, 99 \%$ of plantar pressure signal frequencies below $15 \mathrm{~Hz}$, so the need to design a low-pass filter circuit to filter mixed in plantar pressure signal clutter and noise. The 8-channel sensor signal were sent to the eight voltage selector switch CD4051, then separately voltage amplified and low-pass filtering process, The signals were sent the microprocessor MSP430 with A / D interface to A / D conversion, and the data was stored in the buffer zone, Then the every full 8-channel signal acquisition by nRF905 radio transmission, and by nRF905 radio reception, after MSP430 microprocessor treatment, These 8-channel signal was from the TTL level to USB level by FT232RL, Via USB interface to transfer to the PC, Application of LabVIEW virtual instrument development tools wrote software to achieve the signal display, storage and analysis. Insole plantar pressure measurement system hardware block diagram shown in Figure 2.

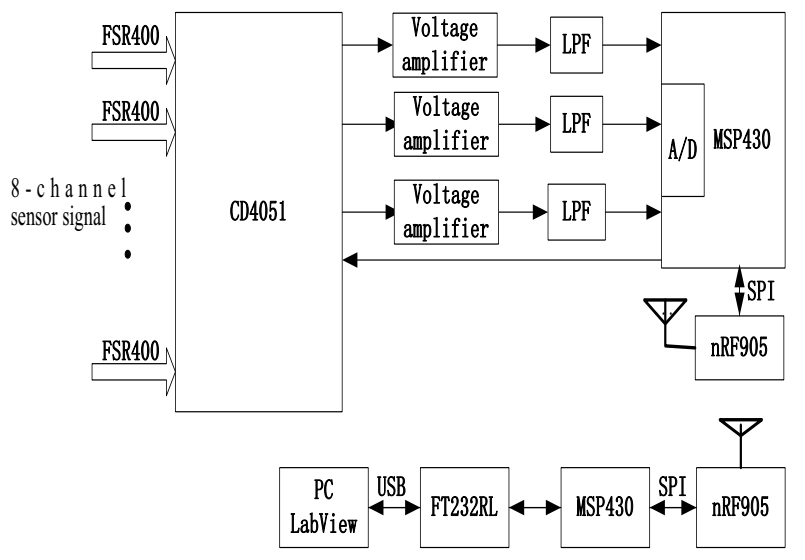

Figure 2. Insole plantar pressure measurement system hardware block diagram

Wireless transceiver module nRF905 built a complete communication protocol and CRC check circuit, and from on-board hardware automatically Manchester encoding / decoding, Just to complete all of the radio transmission through the SPI interface. Use of wireless communications technology, the collected signal was transmitted to the computer for processing, The sets of the system is easy to carry, flexible, saving transmission wire and avoiding the interference wire leaving the data transmission errors occur.

\subsection{Data Acquisition HMI}

Data Acquisition HMI was made by LabVIEW software. LabVIEW is virtual instrument integration environment in laboratory, It uses the structure of the graphics mode to build the program code, taking full advantage of developers familiar icons, terms and concepts, providing a portable way to test equipment programming and data acquisition systems, making the program preparation process intuitive.

First, according to the design requirements, the serial port was initialized, set the serial port was set and the baud rate was set to $9600 \mathrm{~b} / \mathrm{s}, 8$ data bits, 1 stop bit, no parity. Second, the control system was set to start/pause/resume/stop/sampling interval frequency; Setting pressure threshold, when the pressure is greater than this value, the corresponding channel curve becomes green, setting the display area Y-axis display range; Setting data save path, note that the file must be in “.txt” as suffix.

PC sends a start command to the microprocessor "Start", After the microprocessor receives, sends a signal "DSR" of "ready to send" to the PC, and wait for the PC feedback signal "R" of "ready to receive", and then start to send a frame of data, the transmission is completed, return to the initial point, waiting for the next start command. After the PC receives the data, first by "String to Byte Array" command to convert the string into an array, and by measuring the file command data to the "pressure data .txt" file is saved in the appropriate folder while displaying real-time data 8 channels, each channel can display the current value of the measured data. Data Acquisition HMI shown in Figure 3. 


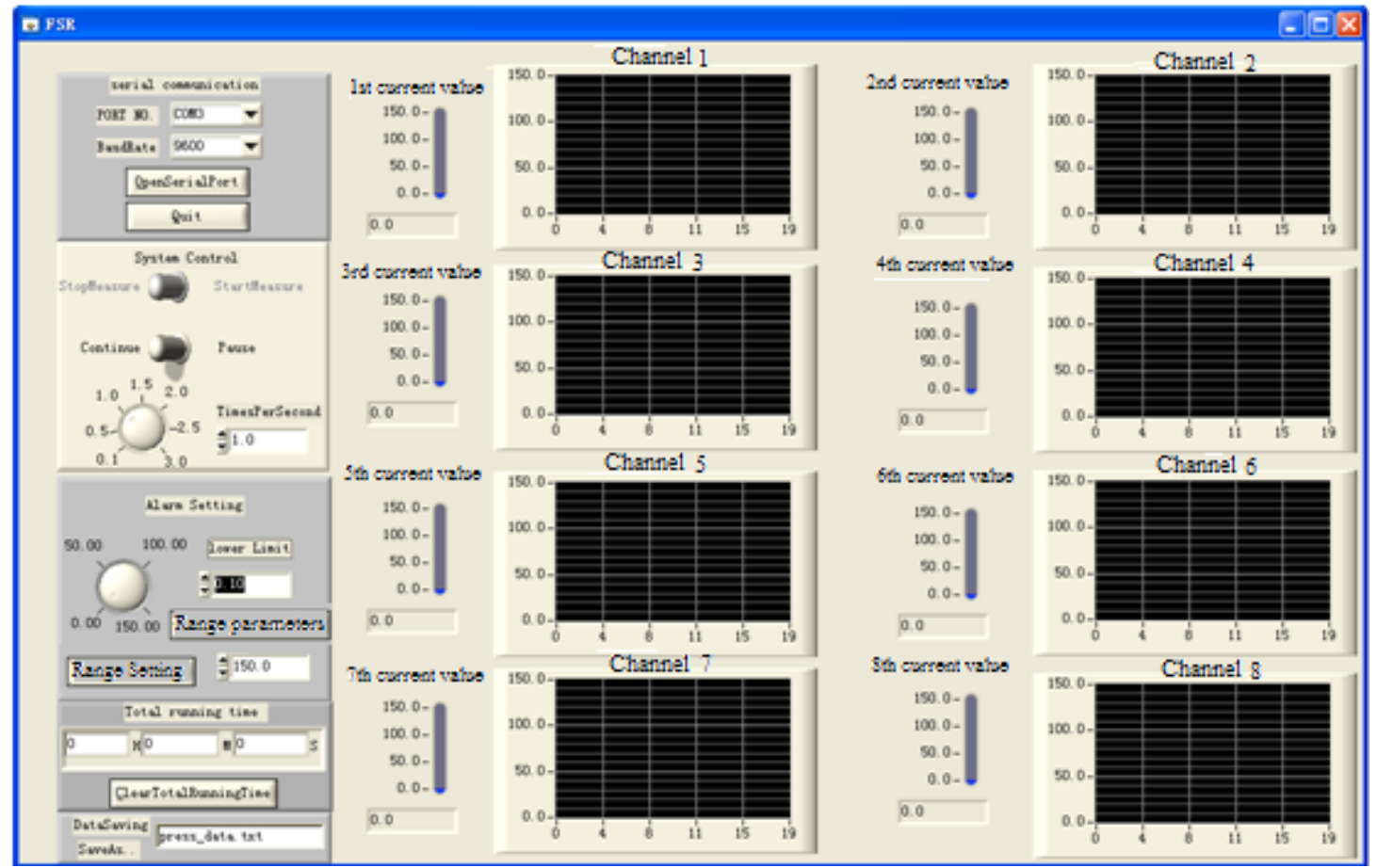

Figure 3. Data Acquisition HMI

\section{Data Analysis and Processing}

\subsection{Membership Function}

Using the insole plantar pressure gathering system above to process 67 cases of each normal walking gait cycle corresponding to pressure sensor values, statistical analysis obtain correspondence between each gait cycle and pressure sensor values relative size distribution patterns. The average peak experimentally measured 67 cases of normal walking process pressure sensor as shown in Table 1.

Table 1. The normal district average peak plantar pressure data $(\bar{x} \pm s) N / \mathrm{cm}^{2}$

\begin{tabular}{|c|c|}
\hline District & Data \\
\hline Heel & $35.85 \pm 1.23$ \\
\hline Arch & $5.50 \pm 0.45$ \\
\hline 1st metatarsal & $0.93 \pm 0.48$ \\
\hline Second metatarsal & $12.24 \pm 0.63$ \\
\hline 3rd metatarsal & $30.67 \pm 1.68$ \\
\hline 4th metatarsal & $31.44 \pm 1.13$ \\
\hline 5th metatarsal & $12.87 \pm 0.74$ \\
\hline 1st toe & $25.42 \pm 0.57$ \\
\hline
\end{tabular}

The process of between twice land of the same foot heel is a gait cycle. A gait cycle can be divided into the swing phase and the stance phase. When the swing phase, leg off the ground and in the air, swinging forward. The stance phase with the lower limbs in contact with the ground to withstand the ground reaction force and a role in stabilizing the body. In the stance phase, can be divided into five stages (five kinds of distribution patterns):( 1) Initial Contact, (2) Loading Response, (3) Mid Stance, (4) Heel Rise, (5) Toe Contac ${ }^{\mathrm{t}[3][4]}$.

Due to the experimental subject's weight, height and other differences, the sensor data for the individual differences in subjects is different, so if only thinking of the specific sensor measurements, it is difficult to judge the subjects in which stance phase, so using fuzzy mathematical to process the experimental data, and using the membership function to represent the relative size of the measured sensor data. For each measurement sensor, the corresponding membership functions such as formula (1):

$$
\mu=\frac{1}{\pi} \arctan \left[a\left(x-x_{0}\right)\right]+\frac{1}{2}
$$

Where $x_{0}$ is the sensor values subject standing still, $a$ is as the adjustment factor, the value of $a$ is the greater, the membership function ${ }^{\mu}$ corresponding to the curve is steeper. 


\subsection{Plantar Pressure Distribution Pattern Recognition}

Fuzzy Recognition is often used in two ways: one is the maximum membership degree principle, also called direct method, which is mainly used to identify a single target; The other is closeness principle, also known as the indirect method, which is generally used for the cluster target identification. Closeness is the proximity of two fuzzy sets of metrics. In this paper, the principle of elective near close degree of grid to identify the subjects in which the stance phase (distribution mode).

$$
\text { Let } A_{i}(i=1,2, \cdots, n) \text { be a fuzzy set of theory }
$$

domain, constitutes a standard model library, it is to be a recognized fuzzy subset of domain U. If there is

Closeness

$$
N\left(B, A_{j}\right)=\max \left\{N\left(B, A_{1}\right), N\left(B, A_{2}\right), \ldots, N\left(B, A_{n}\right)\right\}
$$

, Called $B$ and $A_{j}$ are the most close, That is considered

$B$ to be $A_{j}$ models. The grid closeness between $A_{i}$ and $\mathrm{B}$ is

$$
N\left(A_{i}, B\right)=\frac{1}{2}\left[A_{i} \bullet B+\left(1-A_{i} \odot B\right)\right]
$$

Among them, $A_{i} \bullet B$ is called the inner product of $A_{i}$ and B, $A \bullet B=\vee\left[u_{A} \wedge u_{B}\right] ; A_{i} \odot B$ is called outer $\operatorname{product~of~}^{A_{i}}$ and B, $A \odot B=\wedge\left[u_{A} \vee u_{B}\right]$.

The 5 stages of the stance phase are corresponding to five pressure distribution patterns. According to these five kinds of distribution patterns, the plantar pressure data of the 67 cases subjects are divided into five categories, fuzzy them and getting five sample sets, each element of these sample sets is 8-dimensional, is corresponding to fuzzy membership of 8 sensors. Each sample set is statistical and averaging, obtaining 5 standard distribution model $\left\{A_{1}, A_{2}, A_{3}, A_{4}, A_{5}\right\}$.

During walking, 8 sensor is placed on the foot to collect of the plantar pressure data, these data can be converted into membership, characterizing pressure value relative size. Let $B=\left\{b_{1}, b_{2}, \bullet \bullet, b_{n}\right\}_{\text {represent a }}$ sample set of sample, $b_{i}$ is a element of set, each element consists of eight data, to the ith element there has $b_{i}=\left\{\mu_{1}, \mu_{2}, \cdots, \mu_{8}\right\}, \mu_{i}$ is pressure sensor corresponding membership value after conversion. Specific distribution pattern is determined as follows:(1) Collect data plantar pressure during walking, get to $b_{i}=\left\{\mu_{1}, \mu_{2}, \bullet \bullet, \mu_{8}\right\}$, determine the tolerance $\varepsilon$;

Calculate $N_{j}\left(A_{j}, b_{i}\right)=\frac{1}{2}\left[A_{j} \circ b_{i}+\left(1-A_{j} \otimes b_{i}\right)\right]$, $A_{j} \in\left\{A_{1}, A_{2}, A_{3}, A_{4}, A_{5}\right\}$; (3) If $N_{j} \leq \varepsilon$, then $b_{i}=A_{j}$

\section{Simulation and Analysis}

In order to verify the effectiveness of this method, we have compiled a handler in Matlab platform, added 15 new subjects, collected in plantar pressure data when they were five kinds of stance phase, substituted the data to the program and got automatic recognition results as shown in Table 2.

Table 2. The algorithm identifies results

\begin{tabular}{|l|c|c|}
\hline \multirow{3}{*}{$\begin{array}{c}\text { Identification } \\
\text { Number }\end{array}$} & Initial Contact & 13 \\
\cline { 2 - 3 } & Loading Response & 12 \\
\cline { 2 - 3 } & Mid Stance & 13 \\
\cline { 2 - 3 } & Heel Rise & 11 \\
\cline { 2 - 3 } & Toe Contact & 13 \\
\hline Number 15 & The average recognition rate & $82.6 \%$ \\
\hline
\end{tabular}

From the experiment, an average recognition rate was $82.6 \%$, we can see the method on human plantar pressure distribution pattern has better recognition performance.

Because plantar pressure distribution pattern and the calculation of five standard distribution models

$$
\left\{A_{1}, A_{2}, A_{3}, A_{4}, A_{5}\right\} \text { are on the basis of statistical data, }
$$

sample number is insufficient that affects to increase the recognition rate. It is necessary to improve sample 
number and increase the sample diversity in the follow-up study.

\section{References}

[1].Meng Chen, Bufu Huang, ka Keung Lee, etal. An Intelligent Shoe-Intergrated System for Plantar Pressure Measurement. Processings of the 2006 IEEE

International Conference on Robotics and Biomimetics, 2006:416-421.

[2].Godi M, Turcato A M, Schieppati M, et al. Test-retest reliability of an insole plantar pressure system to assess gait along linear and curved trajectories[J]. Journal of Neuroengineering \& Rehabilitation, 2014, 11(1):1-8.

[3].Pauk J, Daunoraviciene K, Ihnatouski M, et al. Analysis of the plantar pressure distribution in children with foot deformities.[J]. Acta of Bioengineering \& Biomechanics, 2010, 12(1):29-34.
[4]. Mao B Y, Jia X W, Zheng F R. Research on the Human Foot Pressure Distribution of Walking and Standing[J]. Orthopedic Journal of China, 2002.

\section{Fund Project}

1.Hainan social development science and technology projects: Research of the human plantar pressure characteristics Identification based on fuzzy theory(Item Number: 2015SF33) .

2.Hainan Natural Science Fund: Biogeography Optimization Algorithm Based on Multi-objective to Solve Biological Motif and Discovery Problem(Item Number: 20156226).

3.Hainan Natural Science Fund: Key Technology Research of Smart Video Object Segmentation and Tracking Based on DRLSE(Item Number: 20156228). 